\title{
A central limit theorem for the stochastic heat equation
}

\section{Huang, Jingyu}

2020-12

Huang , J , Nualart , D \& Viitasaari , L 2020 , ' A central limit theorem for the stochastic heat equation ' , Stochastic Processes and Their Applications , vol. 130 , no. 12 , pp. 7170-7184 . https://doi.org/10.1016/

http://hdl.handle.net/10138/335658

https://doi.org/10.1016/j.spa.2020.07.010

cc_by_nc_nd

acceptedVersion

Downloaded from Helda, University of Helsinki institutional repository.

This is an electronic reprint of the original article.

This reprint may differ from the original in pagination and typographic detail.

Please cite the original version. 


\title{
A CENTRAL LIMIT THEOREM FOR THE STOCHASTIC HEAT EQUATION
}

\author{
JINGYU HUANG, DAVID NUALART, AND LAURI VIITASAARI
}

\begin{abstract}
We consider the one-dimensional stochastic heat equation driven by a multiplicative space-time white noise. We show that the spatial integral of the solution from $-R$ to $R$ converges in total variance distance to a standard normal distribution as $R$ tends to infinity, after renormalization. We also show a functional version of this central limit theorem.
\end{abstract}

Mathematics Subject Classifications (2010): 60H15, 60H07, 60G15, 60F05.

Keywords: Stochastic heat equation, central limit theorem, Malliavin calculus, Stein's method.

\section{Introduction}

We consider the one-dimensional stochastic heat equation

$$
\frac{\partial u}{\partial t}=\frac{1}{2} \Delta u+\sigma(u) \dot{W}
$$

on $\mathbb{R}_{+} \times \mathbb{R}$, where $\dot{W}$ is a space-time Gaussian white noise, with initial condition $u_{0}(x)=1$. The coefficient $\sigma$ is a Lipschitz function.

It is well-known (see, for instance, [10]) that this equation has a unique mild solution, which is adapted to the filtration generated by $W$, such that $\mathbb{E}\left[|u(t, x)|^{2}\right]<\infty$ and it satisfies the evolution equation

$$
u(t, x)=1+\int_{0}^{t} \int_{\mathbb{R}} p_{t-s}(x-y) \sigma(u(s, y)) W(d s, d y),
$$

where in the right hand side the stochastic integral is in the sense of Walsh, and $p_{t}(x)=(2 \pi t)^{-1 / 2} e^{-x^{2} /(2 t)}$ is the heat kernel.

In this paper we are interested in the asymptotic behavior as $R$ tends to infinity of the quantity

$$
F_{R}(t):=\frac{1}{\sigma_{R}}\left(\int_{-R}^{R} u(t, x) d x-2 R\right),
$$

D. Nualart is supported by NSF Grant DMS 1811181. 
where $R>0, u(t, x)$ is the solution to (1.1) and $\sigma_{R}^{2}=\operatorname{Var}\left(\int_{-R}^{R} u(t, x) d x\right)$.

From equation (1.2) and the properties of the heat kernel, it follows that the solution to equation (1.1) satisfies a localization property. This means that, for any fixed $t>0$, the random variable $u(t, x)$ essentially depends on the noise in a small interval $[x-\epsilon, x+\epsilon]$. This property has been extensively used in the literature, see for example, [2, 3, 4,

In particular, for the parabolic Anderson model $(\sigma(u)=u)$, it is shown in [3] that for each fixed $t>0$, almost surely, the solution $u(t, x)$ develops high peaks along the $x$-axis. More precisely, it holds that, almost surely

$$
0<\limsup _{R \rightarrow \infty} \frac{\max _{|x| \leq R} \log u(t, x)}{(\log R)^{2 / 3}}<\infty .
$$

The basic idea in [3] to show this result is that one can define a "localized version" of equation (1.1) with solution $U(t, x)$, such that, whenever $x_{i}$ and $x_{j} \in \mathbb{R}$ are far apart for $i \neq j, U\left(t, x_{i}\right), i=0, \pm 1, \pm 2, \ldots$, are i.i.d. random variables, and also $U(t, x)$ and $u(t, x)$ are close in certain sense. Since a rare event (high peak in this case) will happen with high probability if there are enough independent random variables, i.e., $U\left(t, x_{i}\right), i=0, \pm 1, \pm 2, \ldots$, one can see that $u(t, x)$, which is close to $U(t, x)$, also develops high peaks.

Following this idea, the spatial integral $\int_{R}^{R} u(t, x) d x$ is similar to a sum of i.i.d. random variables and we expect that certain central limit theorem holds in this case. To be more precise, our first result is the following quantitative central limit theorem:

Theorem 1.1. Suppose that $u(t, x)$ is the mild solution to equation (1.1) and let $F_{R}(t)$ be given by (1.3). Suppose that $\sigma_{R}>0$. Let $d_{T V}$ denote the total variation distance and let $Z \sim \mathcal{N}(0,1)$. Then there exists a constant $C$, depending only on $t$, such that

$$
d_{T V}\left(F_{R}(t), Z\right) \leq \frac{C}{\sqrt{R}}
$$

Remark. Condition $\sigma(1) \neq 0$ guarantees that $\sigma_{R}>0$. Notice that this condition is not necessary. Taking into account that $\sigma_{R}=0$ implies

$$
\int_{0}^{t} \int_{\mathbb{R}} \mathbb{E}\left(\sigma^{2}(u(s, y))\right)\left(\int_{-R}^{R} p_{t-s}(x-y) d x\right)^{2} d y d s=0,
$$

a sufficient condition would be that $\sigma(u(s, y))$ is not identically zero on $[0, t] \times \mathbb{R}$ with positive probability. 
We will show (see Proposition (3.1) ) that the variance $\sigma_{R}^{2}$ satisfies

$$
\lim _{R \rightarrow \infty} \frac{\sigma_{R}^{2}}{R}=2 \int_{0}^{t} \xi(s) d s
$$

where $\xi(s)=\mathbb{E}\left[\sigma(u(s, y))^{2}\right]$. It turns out that $\mathbb{E}\left[\sigma(u(s, y))^{2}\right]$ does not depend on $y \in \mathbb{R}$ and is bounded on compact intervals. Then, we also prove the following functional version of Theorem 1.1 with a normalization by $1 / \sqrt{R}$.

Theorem 1.2. Suppose that $u(t, x)$ is the mild solution to equation (1.1). Set $\xi(s)=\mathbb{E}\left[\sigma(u(s, y))^{2}\right], s \geq 0$. Then, for any $T>0$,

$$
\left(\frac{1}{\sqrt{R}}\left(\int_{-R}^{R} u(t, x) d x-2 R\right)\right)_{t \in[0, T]} \rightarrow\left(\int_{0}^{t} \sqrt{2 \xi(s)} d B_{s}\right)_{t \in[0, T]},
$$

as $R$ tends to infinity, where $B$ is a Brownian motion and the convergence is in law on the space of continuous functions $C([0, T])$.

Theorem 1.1 is proved using a combination of Stein's method for normal approximations and Malliavin calculus, following the ideas introduced by Nourdin and Peccati in [6]. An innovative aspect of our methodology is to use the representation of $F_{R}(t)$ as a divergence, taking into account that the Itô-Walsh integral is a particular case of the Skorohod integral.

The rest of the paper is organized as follows. In Section 2 we recall some preliminaries on Malliavin calculus and Stein's method. Sections 3 and 4 are devoted to the proofs of our main theorems. We put one technical lemma into the appendix.

\section{Preliminaries}

Let us first introduce the white noise on $\mathbb{R}_{+} \times \mathbb{R}$. We denote by $\mathcal{B}_{b}\left(\mathbb{R}_{+} \times\right.$ $\mathbb{R})$ the collection of Borel sets $A \subset \mathbb{R}_{+} \times \mathbb{R}$ with finite Lebesgue measure, denoted by $|A|$. Consider a centered Gaussian family of random variables $W=\left\{W(A), A \in \mathcal{B}_{b}\right\}$, defined in a complete probability space $(\Omega, \mathcal{F}, P)$, with covariance

$$
\mathbb{E}[W(A) W(B)]=|A \cap B| .
$$

For any $t \geq 0$, we denote by $\mathcal{F}_{t}$ the $\sigma$-field generated by the random variables $\left\{W([0, s] \times A): 0 \leq s \leq t, A \in \mathcal{B}_{b}(\mathbb{R})\right\}$. As proved in [10], for any adapted random field $\left\{X(s, y),(s, y) \in \mathbb{R}_{+} \times \mathbb{R}\right\}$ that is jointly measurable and

$$
\int_{0}^{\infty} \int_{\mathbb{R}} \mathbb{E}\left[X(s, y)^{2}\right] d y d s<\infty
$$


the following stochastic integral

$$
\int_{0}^{\infty} \int_{\mathbb{R}} X(s, y) W(d s, d y)
$$

is well-defined.

The proof of the main theorems relies on Malliavin calculus and Stein's method. Next we will introduce the basic elements of these methodologies.

2.1. Malliavin calculus. In this subsection we recall some basic facts on the Malliavin calculus associated with $W$. We refer to [7] for a detailed account on the Malliavin calculus with respect to a Gaussian process. Consider the Hilbert space $\mathfrak{H}=L^{2}\left(\mathbb{R}_{+} \times \mathbb{R}\right)$. The Wiener integral

$$
W(h)=\int_{0}^{\infty} \int_{\mathbb{R}} h(t, x) W(d t, d x)
$$

provides an isometry between the Hilbert space $\mathfrak{H}$ and $L^{2}(\Omega)$. In this sense $\{W(h), h \in \mathfrak{H}\}$ is an isonormal Gaussian process.

Denote by $C_{p}^{\infty}\left(\mathbb{R}^{n}\right)$ the space of smooth functions with all their partial derivatives having at most polynomial growth at infinity. Let $\mathcal{S}$ be the space of simple random variables of the form

$$
F=f\left(W\left(h_{1}\right), \ldots, W\left(h_{n}\right)\right)
$$

for $f \in C_{p}^{\infty}\left(\mathbb{R}^{n}\right)$ and $h_{i} \in \mathfrak{H}, 1 \leq i \leq n$. Then $D F$ is the $\mathfrak{H}$-valued random variable defined by

$$
D F=\sum_{i=1}^{n} \frac{\partial f}{\partial x_{i}}\left(W\left(h_{1}\right), \ldots, W\left(h_{n}\right)\right) h_{i} .
$$

The derivative operator $D$ is a closable operator from $L^{p}(\Omega)$ into $L^{p}(\Omega ; \mathfrak{H})$ for any $p \geq 1$. For any $p \geq 1$, let $\mathbb{D}^{1, p}$ be the completion of $\mathcal{S}$ with respect to the norm

$$
\|F\|_{1, p}=\left(\mathbb{E}|F|^{p}+\mathbb{E}\left(\|D F\|_{\mathfrak{H}}^{p}\right)^{1 / p} .\right.
$$

We denote by $\delta$ the adjoint of the derivative operator given by the duality formula

$$
\mathbb{E}(\delta(u) F)=\mathbb{E}\left(\langle u, D F\rangle_{\mathfrak{H}}\right)
$$

for any $F \in \mathbb{D}^{1,2}$, and any $u \in L^{2}(\Omega ; \mathfrak{H})$ in the domain of $\delta$, denoted by Dom $\delta$. The operator $\delta$ is also called the Skorohod integral because in the case of the Brownian motion, it coincides with an extension of the Itô integral introduced by Skorohod (see [5, 8]). More generally, in the context of the space-time white 
noise $W$, any adapted random field $X$ which is jointly measurable and satisfies (2.1) belongs to the domain of $\delta$ and $\delta(X)$ coincides with the Walsh integral:

$$
\delta(X)=\int_{0}^{\infty} \int_{\mathbb{R}} X(s, y) W(d s, d y) .
$$

As a consequence, the mild equation (1.2) can also be written as

$$
u(t, x)=1+\delta\left(p_{t-.}(x-*) u(\cdot, *)\right) .
$$

It is known that for any $(t, x)$ the solution $u(t, x)$ of equation (1.1) belongs to $\mathbb{D}^{1, p}$ for any $p \geq 2$ and the derivative satisfies the following linear stochastic integral differential equation for $t \geq s$,

$$
\begin{aligned}
D_{s, y} u(t, x)= & p_{t-s}(x-y) \sigma(u(s, y)) \\
& +\int_{s}^{t} \int_{\mathbb{R}} p_{t-r}(x-z) \Sigma(r, z) D_{s, y} u(r, z) W(d r, d z),
\end{aligned}
$$

where $\Sigma(r, z)$ is an adapted process, bounded by the Lipschitz constant of $\sigma$. If $\sigma$ is continuously differentiable, then $\Sigma(r, z)=\sigma^{\prime}(u(r, z))$. This result is proved in Proposition 2.4.4 of [7] in the case of Dirichlet boundary conditions on [0,1] and the proof can be easily extended to equations on $\mathbb{R}$. We also refer to [1, 9] for additional references where this result is used when $\sigma$ is continuously differentiable.

2.2. Stein's method. Stein's method is a probabilistic technique which allows one to measure the distance between a probability distribution and normal distribution. The total variance distance between two random variables $F$ and $G$ is defined by

$$
d_{T V}(F, G):=\sup _{B \in \mathcal{B}(\mathbb{R})}|P(F \in B)-P(G \in B)|,
$$

where $\mathcal{B}(\mathbb{R})$ is the collection of all Borel sets in $\mathbb{R}$. We point out that $d_{T V}(F, G)$ only depends on the laws of $F$ and $G$ and it defines a metric on the set of probability measures on $\mathbb{R}$.

The following theorem provides an upper bound for the total variation distance between any random variable and a random variable with standard normal distribution.

Theorem 2.1. For $Z \sim \mathcal{N}(0,1)$ and for any random variable $F$,

$$
d_{T V}(F, Z) \leq \sup _{f \in \mathscr{F}_{T V}}\left|\mathbb{E}\left[f^{\prime}(F)\right]-\mathbb{E}[F f(F)]\right|
$$

where $\mathscr{F}_{T V}$ is the class of continuously differentiable functions $f$ such that $\|f\|_{\infty} \leq \sqrt{\pi / 2}$ and $\left\|f^{\prime}\right\|_{\infty} \leq 2$. 
See [6] for a proof of this theorem. Theorem 2.1] can be combined with Malliavin calculus to get the following estimate.

Proposition 2.2. Let $F=\delta(v)$ for some $\mathfrak{H}$-valued random variable $v$ which belongs to Dom $\delta$. Assume $\mathbb{E}\left[F^{2}\right]=1$ and $F \in \mathbb{D}^{1,2}$. Let $Z \sim \mathcal{N}(0,1)$. Then we have

$$
d_{T V}(F, Z) \leq 2 \sqrt{\operatorname{Var}\langle D F, v\rangle_{\mathfrak{H}}} .
$$

Proof. By our assumption on $F$, we have

$$
\begin{aligned}
\mathbb{E}[F f(F)] & =\mathbb{E}[\delta(v) f(F)]=\mathbb{E}\langle v, D[f(F)]\rangle_{\mathfrak{H}} \\
& =\mathbb{E}\left\langle v, f^{\prime}(F) D F\right\rangle_{\mathfrak{H})}=\mathbb{E}\left(f^{\prime}(F)\langle v, D F\rangle_{\mathfrak{H}}\right) .
\end{aligned}
$$

Thus, by Theorem 2.1 ,

$$
\begin{aligned}
d_{T V}(F, Z) & \leq \sup _{f \in \mathscr{F}_{T V}}\left|\mathbb{E}\left[f^{\prime}(F)-F f(F)\right]\right| \\
& =\sup _{f \in \mathscr{F}_{T V}}\left|\mathbb{E}\left[f^{\prime}(F)\left(1-\langle D F, v\rangle_{\mathfrak{H}}\right)\right]\right| \\
& \leq 2 \mathbb{E}\left(\left|1-\langle D F, v\rangle_{\mathfrak{H}}\right|\right) \\
& \leq 2 \sqrt{\operatorname{Var}\langle D F, v\rangle_{\mathfrak{H}}},
\end{aligned}
$$

where the last step follows from Cauchy-Schwarz inequality, (2.3) and

$$
\mathbb{E}\left(\langle D F, v\rangle_{\mathfrak{H}}\right)=\mathbb{E}[F \delta(v)]=\mathbb{E}\left(F^{2}\right)=1 .
$$

In proving Theorem 1.2 we also need the following proposition, which is a generalization of Theorem 6.1.2 in [6].

Proposition 2.3. Let $F=\left(F^{(1)}, \ldots, F^{(m)}\right)$ be a random vector such that $F^{(i)}=\delta\left(v^{(i)}\right)$ for $v^{(i)} \in \operatorname{Dom} \delta, i=1, \ldots, m$. Assume $F^{(i)} \in \mathbb{D}^{1,2}$ for $i=$ $1, \ldots, m$. Let $Z$ be an $m$-dimensional Gaussian centered vector with covariance matrix $\left(C_{i, j}\right)_{1 \leq i, j \leq m}$. For any $C^{2}$ function $h: \mathbb{R}^{m} \rightarrow \mathbb{R}$ with bounded second partial derivatives, we have

$$
\left|\mathbb{E} h\left(F_{R}\right)-\mathbb{E} h(Z)\right| \leq \frac{1}{2}\left\|h^{\prime \prime}\right\|_{\infty} \sqrt{\sum_{i, j=1}^{m} \mathbb{E}\left[\left(C_{i, j}-\left\langle D F^{(i)}, v^{(j)}\right\rangle_{\mathfrak{H}}\right)^{2}\right]}
$$

where

$$
\left\|h^{\prime \prime}\right\|_{\infty}=\max _{1 \leq i, j \leq m} \sup _{x \in \mathbb{R}^{m}}\left|\frac{\partial^{2} h}{\partial x_{i} \partial x_{j}}(x)\right|
$$


Proof. The proof will follow the same ideas as those in the proof of Theorem 6.1.2 in [6]. Without loss of generality, we may assume that $Z$ and $F$ are independent. Let

$$
\Phi(t)=\mathbb{E}[h(\sqrt{1-t} F+\sqrt{t} Z)]
$$

Then

with

$$
\mathbb{E}[h(Z)]-\mathbb{E}[h(F)]=\Phi(1)-\Phi(0)=\int_{0}^{1} \Phi^{\prime}(t) d t
$$

$$
\Phi^{\prime}(t)=\sum_{i=1}^{m} \mathbb{E}\left(\frac{\partial h}{\partial x_{i}}(\sqrt{1-t} F+\sqrt{t} Z)\left[\frac{1}{2 \sqrt{t}} Z^{(i)}-\frac{1}{2} \frac{1}{\sqrt{1-t}} F^{(i)}\right]\right) .
$$

The above expression is a sum of two expectations. For the first expectation, the proof of Theorem 6.1.2 in [6] already yields that

$\mathbb{E}\left(\frac{\partial h}{\partial x_{i}}(\sqrt{1-t} F+\sqrt{t} Z) Z^{(i)}\right)=\sqrt{t} \sum_{j=1}^{m} C_{i, j} \mathbb{E}\left(\frac{\partial^{2} h}{\partial x_{i} \partial x_{j}}(\sqrt{1-t} F+\sqrt{t} Z)\right)$.

For the second expectation, let $\mathbb{E}_{F}$ be the expectation conditioned on $Z$, then we have

$$
\begin{aligned}
& \mathbb{E}\left(\frac{\partial h}{\partial x_{i}}(\sqrt{1-t} F+\sqrt{t} Z) F^{(i)}\right) \\
= & \mathbb{E}_{F}\left(\frac{\partial h}{\partial x_{i}}(\sqrt{1-t} F+\sqrt{t} Z) \delta\left(v^{(i)}\right)\right) \\
= & \mathbb{E}_{F}\left(\left\langle D \frac{\partial h}{\partial x_{i}}(\sqrt{1-t} F+\sqrt{t} Z), v^{(i)}\right\rangle_{\mathfrak{H}}\right) \\
= & \sqrt{1-t} \sum_{j=1}^{m} \mathbb{E}\left(\frac{\partial^{2} h}{\partial x_{i} \partial x_{j}}(\sqrt{1-t} F+\sqrt{t} Z)\left\langle D F^{(j)}, v^{(i)}\right\rangle_{\mathfrak{H}}\right) .
\end{aligned}
$$

Finally, combining the above calculation with (2.9) and (2.10) with an application of Cauchy-Schwarz inequality completes the proof.

\section{Proof of Theorem 1.1}

We begin by computing the asymptotic covariance of $F_{R}(t)$ as $R$ tends to infinity. This will be also relevant in the proof of Theorem 1.2 .

Proposition 3.1. Denote $\xi(r)=\mathbb{E}\left[\sigma(u(r, x))^{2}\right]$ and set

$$
G_{R}(t)=\int_{-R}^{R} u(t, x) d x-2 R
$$


Then, for any $s, t \geq 0$,

$$
\lim _{R \rightarrow \infty} \frac{1}{R} \operatorname{Cov}\left(G_{R}(t), G_{R}(s)\right)=2 \int_{0}^{s \wedge t} \xi(r) d r .
$$

Proof. Thanks to the Itô isometry we have

$$
\begin{aligned}
\mathbb{E}\left[u(t, x) u\left(s, x^{\prime}\right)\right] & =1+\int_{0}^{s \wedge t} \int_{\mathbb{R}} p_{t-r}(x-y) p_{s-r}\left(x^{\prime}-y\right) \mathbb{E}\left[\sigma(u(r, y))^{2}\right] d y d r \\
& =1+\int_{0}^{s \wedge t} \int_{\mathbb{R}} \xi(r) p_{t-r}\left(x^{\prime}-y\right) p_{s-r}(x-y) d y d r \\
& =1+\int_{0}^{s \wedge t} \xi(r) p_{t+s-2 r}\left(x-x^{\prime}\right) d r
\end{aligned}
$$

where in the last line we have used the semigroup property

$$
\int_{\mathbb{R}} p_{t}\left(x^{\prime}-y\right) p_{s}(y-x) d y=p_{t+s}\left(x^{\prime}-x\right) .
$$

Since

$$
\mathbb{E}\left(\int_{-R}^{R} u(t, x) d x\right)=2 R
$$

we obtain

$$
\begin{aligned}
\operatorname{Cov}\left(G_{R}(t), G_{R}(s)\right) & =\int_{-R}^{R} \int_{-R}^{R} \int_{0}^{s \wedge t} \xi(r) p_{t+s-2 r}\left(x-x^{\prime}\right) d r d x d x^{\prime} \\
& =2 \int_{0}^{s \wedge t} \xi(r) \int_{0}^{2 R} p_{t+s-2 r}(z)(2 R-z) d z d r
\end{aligned}
$$

As a consequence,

$$
\begin{aligned}
\lim _{R \rightarrow \infty} \frac{1}{R} \operatorname{Cov}\left(G_{R}(t), G_{R}(s)\right) & =\lim _{R \rightarrow \infty} 2 \int_{0}^{s \wedge t} \xi(r) \int_{0}^{2 R} p_{t+s-2 r}(z)\left(2-\frac{z}{R}\right) d z d r \\
& =2 \int_{0}^{s \wedge t} \xi(r) d r .
\end{aligned}
$$

This concludes the proof.

We are now ready to prove Theorem 1.1.

Proof of Theorem 1.1. By Proposition 2.2, we know that for any $F \in \mathbb{D}^{1,2}$ such that $\mathbb{E}\left(F^{2}\right)=1$ and $F=\delta(v)$,

$$
d_{T V}(F, Z) \leq 2 \sqrt{\operatorname{Var}\left(\langle D F, v\rangle_{\mathfrak{H}}\right)},
$$


where $v$ is such that $F=\delta(v)$. Recall that in our case we have, applying Fubini's theorem,

$$
\begin{aligned}
F_{R}(t) & =\frac{1}{\sigma_{R}}\left(\int_{-R}^{R} u(t, x) d x-2 R\right) \\
& =\frac{1}{\sigma_{R}}\left(\int_{-R}^{R} \int_{0}^{t} \int_{\mathbb{R}} p_{t-s}(x-y) \sigma(u(s, y)) W(d s, d y) d x\right) \\
& =\int_{0}^{t} \int_{\mathbb{R}}\left(\frac{1}{\sigma_{R}} \int_{-R}^{R} p_{t-s}(x-y) \sigma(u(s, y)) d x\right) W(d s, d y) .
\end{aligned}
$$

As a consequence, taking into account equation (2.4), we have, for any fixed $t \geq 0, F_{R}(t)=\delta\left(v_{R}\right)$, where

$$
v_{R}(s, y)=\mathbf{1}_{[0, t]}(s) \frac{1}{\sigma_{R}} \int_{-R}^{R} p_{t-s}(x-y) \sigma(u(s, y)) d x .
$$

Moreover,

$$
D_{s, y} F_{R}=\mathbf{1}_{[0, t]}(s) \frac{1}{\sigma_{R}} \int_{-R}^{R} D_{s, y} u(t, x) d x .
$$

Therefore,

$$
\left\langle D F_{R}(t), v_{R}\right\rangle_{\mathfrak{H}}=\frac{1}{\sigma_{R}^{2}} \int_{0}^{t} \int_{\mathbb{R}} \int_{-R}^{R} \int_{-R}^{R} p_{t-s}(x-y) \sigma(u(s, y)) D_{s, y} u\left(t, x^{\prime}\right) d x d x^{\prime} d y d s .
$$

From (2.5), we know that

$$
\begin{aligned}
D_{s, y} u\left(t, x^{\prime}\right)= & p_{t-s}\left(x^{\prime}-y\right) \sigma(u(s, y)) \\
& +\int_{s}^{t} \int_{\mathbb{R}} p_{t-r}\left(x^{\prime}-z\right) \Sigma(r, z) D_{s, y} u(r, z) W(d r, d z),
\end{aligned}
$$

where $\Sigma(r, z)$ is a bounded and adapted random field. This produces the decomposition

$$
\begin{aligned}
\left\langle D F_{R}(t), v_{R}\right\rangle_{\mathfrak{H}}= & \frac{1}{\sigma_{R}^{2}} \int_{0}^{t} \int_{\mathbb{R}}\left(\int_{-R}^{R} p_{t-s}(x-y) d x\right)^{2} \sigma^{2}(u(s, y)) d y d s \\
& +\frac{1}{\sigma_{R}^{2}} \int_{0}^{t} \int_{\mathbb{R}} \int_{-R}^{R} \int_{-R}^{R} p_{t-s}(x-y) \sigma(u(s, y)) \\
& \times\left(\int_{s}^{t} \int_{\mathbb{R}} p_{t-r}(\tilde{x}-z) \Sigma(r, z) D_{s, y} u(r, z) W(d r, d z)\right) d x d \tilde{x} d y d s .
\end{aligned}
$$


Therefore, using that for any process $\Phi=\{\Phi(s), s \in[0, t]\}$ such that $\sqrt{\operatorname{Var}\left(\Phi_{s}\right)}$ is integrable on $[0, t]$, we have

$$
\sqrt{\operatorname{Var}\left(\int_{0}^{t} \Phi_{s} d s\right)} \leq \int_{0}^{t} \sqrt{\operatorname{Var}\left(\Phi_{s}\right)} d s,
$$

we can write

$$
\sqrt{\operatorname{Var}\left(\left\langle D F_{R}(t), v_{R}\right\rangle_{\mathfrak{H}}\right)} \leq A_{1}+A_{2}
$$

where

$$
\begin{aligned}
A_{1}= & \frac{1}{\sigma_{R}^{2}} \int_{0}^{t}\left(\int_{\mathbb{R}^{2}}\left(\int_{-R}^{R} p_{t-s}(x-y) d x\right)^{2}\left(\int_{-R}^{R} p_{t-s}\left(x^{\prime}-y^{\prime}\right) d x^{\prime}\right)^{2}\right. \\
& \left.\times \operatorname{Cov}\left(\sigma^{2}(u(s, y)), \sigma^{2}\left(u\left(s, y^{\prime}\right)\right)\right) d y d y^{\prime}\right)^{\frac{1}{2}} d s
\end{aligned}
$$

and

$$
\begin{aligned}
A_{2}= & \frac{1}{\sigma_{R}^{2}} \int_{0}^{t}\left(\int_{\mathbb{R}^{2}} \int_{[-R, R]^{4}} p_{t-s}(x-y) p_{t-s}\left(x^{\prime}-y^{\prime}\right) \int_{s}^{t} \int_{\mathbb{R}} p_{t-r}(\tilde{x}-z) p_{t-r}\left(\tilde{x}^{\prime}-z\right)\right. \\
& \times \mathbb{E}\left(\sigma(u(s, y)) \sigma\left(u\left(s, y^{\prime}\right)\right) \Sigma^{2}(r, z) D_{s, y} u(r, z) D_{s, y^{\prime}} u(r, z)\right) \\
& \left.\times d z d r d x d x^{\prime} d \tilde{x} d \tilde{x}^{\prime} d y d y^{\prime}\right)^{\frac{1}{2}} d s .
\end{aligned}
$$

The proof will be done in two steps:

Step 1: $\quad$ Let us first estimate the term $A_{2}$. Denote by $L$ the Lipschitz constant of $\sigma$ and let, for $p \geq 2$,

$$
K_{p}(t)=\sup _{0 \leq s \leq t} \sup _{y \in \mathbb{R}}\|\sigma(u(s, y))\|_{p}
$$

Then,

$$
\begin{aligned}
& \left|\mathbb{E}\left(\sigma(u(s, y)) \sigma\left(u\left(s, y^{\prime}\right)\right) \Sigma^{2}(r, z) D_{s, y} u(r, z) D_{s, y^{\prime}} u(r, z)\right)\right| \\
& \leq K_{4}^{2}(t) L^{2}\left\|D_{s, y} u(r, z)\right\|_{4}\left\|D_{s, y^{\prime}} u(r, z)\right\|_{4} .
\end{aligned}
$$

We need to estimate $\left\|D_{s, y} u(r, z)\right\|_{p}$ for any $p \geq 2$. According to (3.2), for any $s \in[0, r]$, applying Burkholder's inequality yields

$$
\begin{aligned}
& \left\|D_{s, y} u(r, z)\right\|_{p} \leq p_{r-s}(z-y) K_{p}(t) \\
& \quad+c_{p}\left(E\left(\left.\left.\left|\int_{s}^{r} \int_{\mathbb{R}} p_{r-r_{1}}^{2}\left(z-z_{1}\right) \Sigma^{2}\left(r_{1}, z_{1}\right)\right| D_{s, y} u\left(r_{1}, z_{1}\right)\right|^{2} d r_{1} d z_{1}\right|^{\frac{p}{2}}\right)\right)^{\frac{1}{p}}
\end{aligned}
$$




$$
\begin{aligned}
\leq & p_{r-s}(z-y) K_{p}(t) \\
& +L c_{p}\left(\int_{s}^{r} \int_{\mathbb{R}} p_{r-r_{1}}^{2}\left(z-z_{1}\right)\left\|D_{s, y} u\left(r_{1}, z_{1}\right)\right\|_{p}^{2} d r_{1} d z_{1}\right)^{\frac{1}{2}},
\end{aligned}
$$

which implies

$$
\begin{aligned}
& \left\|D_{s, y} u(r, z)\right\|_{p}^{2} \leq 2 p_{r-s}^{2}(z-y) K_{p}^{2}(t) \\
& \quad+2 L^{2} c_{p}^{2} \int_{s}^{r} \int_{\mathbb{R}} p_{r-r_{1}}^{2}\left(z-z_{1}\right)\left\|D_{s, y} u\left(r_{1}, z_{1}\right)\right\|_{p}^{2} d z_{1} d r_{1} .
\end{aligned}
$$

By Lemma 5.1, we have the estimate

$$
\left\|D_{s, y} u(r, z)\right\|_{p} \leq C p_{r-s}(z-y),
$$

where the constant $C$ depends on $t$ and $p$.

From (3.4) and Proposition 3.1, we derive the following estimate for the term $A_{2}$ :

$$
\begin{aligned}
A_{2} \leq & \frac{C}{R} \int_{0}^{t}\left(\int_{\mathbb{R}^{2}} \int_{[-R, R]^{4}} \int_{s}^{t} \int_{\mathbb{R}} p_{t-s}(x-y) p_{t-s}\left(x^{\prime}-y^{\prime}\right) p_{t-r}(\tilde{x}-z)\right. \\
& \left.\times p_{t-r}\left(\tilde{x}^{\prime}-z\right) p_{r-s}(z-y) p_{r-s}\left(z-y^{\prime}\right) d z d r d x d x^{\prime} d \tilde{x} d \tilde{x}^{\prime} d y d y^{\prime}\right)^{\frac{1}{2}} d s .
\end{aligned}
$$

Integrating $\tilde{x}, \tilde{x}^{\prime}$ over $\mathbb{R}$, then integrating $y^{\prime}, y$ over $\mathbb{R}$ and using the semigroup property, we obtain

$$
\begin{aligned}
A_{2} & \leq \frac{C}{R} \int_{0}^{t}\left(\int_{[-R, R]^{2}} \int_{s}^{t} \int_{\mathbb{R}} p_{t+r-2 s}(x-z) p_{t+r-2 s}\left(x^{\prime}-z\right) d z d r d x d x^{\prime}\right)^{\frac{1}{2}} d s \\
& \leq \frac{C}{R} \int_{0}^{t}\left(\int_{[-R, R]^{2}} \int_{s}^{t} p_{2 t+2 r-4 s}\left(x-x^{\prime}\right) d r d x d x^{\prime}\right)^{\frac{1}{2}} d s .
\end{aligned}
$$

Finally, integrating $x$ over $\mathbb{R}$ and $x^{\prime}$ over $[-R, R]$, we get

$$
A_{2} \leq \frac{C}{\sqrt{R}}
$$

Step 2: To estimate the term $A_{1}$ we need a bound for the covariance

$$
\operatorname{Cov}\left(\sigma^{2}(u(s, y)), \sigma^{2}\left(u\left(s, y^{\prime}\right)\right)\right) \text {. }
$$

Here, the main idea is to use a version of Clark-Ocone formula for twoparameter processes to write

$$
\sigma^{2}(u(s, y))=\mathbb{E}\left[\sigma^{2}(u(s, y))\right]+\int_{0}^{s} \int_{\mathbb{R}} \mathbb{E}\left[D_{r, z}\left(\sigma^{2}(u(s, y))\right) \mid \mathcal{F}_{r}\right] W(d r, d z)
$$


Then,

$$
\begin{aligned}
& \operatorname{Cov}\left(\sigma^{2}(u(s, y)), \sigma^{2}\left(u\left(s, y^{\prime}\right)\right)\right) \\
& \quad=\int_{0}^{s} \int_{\mathbb{R}} \mathbb{E}\left[\mathbb{E}\left[D_{r, z}\left(\sigma^{2}(u(s, y))\right) \mid \mathcal{F}_{r}\right] \mathbb{E}\left[D_{r, z}\left(\sigma^{2}\left(u\left(s, y^{\prime}\right)\right)\right) \mid \mathcal{F}_{r}\right]\right] d z d r .
\end{aligned}
$$

Applying the chain rule for Lipschitz functions (see [7, Proposition 1.2.4]), we have

$$
D_{r, z}\left(\sigma^{2}(u(s, y))\right)=2 \sigma(u(s, y)) \Sigma(s, y) D_{r, z} u(s, y)
$$

and

$$
\left\|\mathbb{E}\left[D_{r, z}\left(\sigma^{2}(u(s, y))\right) \mid \mathcal{F}_{r}\right]\right\|_{2} \leq 2 K_{4}(t) L\left\|D_{r, z} u(s, y)\right\|_{4} .
$$

Then, using (3.4), we can write

$$
\begin{aligned}
& \left|\operatorname{Cov}\left(\sigma^{2}(u(s, y)), \sigma^{2}\left(u\left(s, y^{\prime}\right)\right)\right)\right| \\
& \leq 4 L^{2} K_{4}^{2}(t) \int_{0}^{s} \int_{\mathbb{R}}\left\|D_{r, z} u(s, y)\right\|_{4}\left\|D_{r, z} u\left(s, y^{\prime}\right)\right\|_{4} d z d r \\
& \leq C \int_{0}^{s} \int_{\mathbb{R}} p_{s-r}(z-y) p_{s-r}\left(z-y^{\prime}\right) d z d r \\
& \quad=C \int_{0}^{s} p_{2 s-2 r}\left(y-y^{\prime}\right) d r .
\end{aligned}
$$

Therefore,

$$
\begin{aligned}
A_{1} \leq & \frac{C}{R} \int_{0}^{t}\left(\int_{\mathbb{R}^{2}}\left(\int_{-R}^{R} p_{t-s}(x-y) d x\right)^{2}\left(\int_{-R}^{R} p_{t-s}\left(x^{\prime}-y^{\prime}\right) d x^{\prime}\right)^{2}\right. \\
& \left.\times \int_{0}^{s} p_{2 s-2 r}\left(y-y^{\prime}\right) d r d y d y^{\prime}\right)^{\frac{1}{2}} d s \\
\leq & \frac{C}{R} \int_{0}^{t}\left(\int_{0}^{s} \int_{\mathbb{R}^{2}} \int_{[-R, R]^{4}} p_{t-s}(x-y) p_{t-s}(\tilde{x}-y) p_{t-s}\left(x^{\prime}-y^{\prime}\right) p_{t-s}\left(\tilde{x}^{\prime}-y^{\prime}\right)\right. \\
& \left.\times p_{2 s-2 r}\left(y-y^{\prime}\right) d x d \tilde{x} d x^{\prime} d \tilde{x}^{\prime} d y d y^{\prime} d r\right)^{\frac{1}{2}} d s .
\end{aligned}
$$

Again, integrate $\tilde{x}$ and $\tilde{x}^{\prime}$ over $\mathbb{R}$, then integrate $y$ and $y^{\prime}$ over $\mathbb{R}$ using the semigroup property, to obtain

$$
A_{1} \leq \frac{C}{R} \int_{0}^{t}\left(\int_{0}^{s} \int_{[-R, R]^{2}} p_{2 t-2 r}\left(x-x^{\prime}\right) d x d x^{\prime} d r\right)^{\frac{1}{2}} d s .
$$


Finally, integrating $x$ over $\mathbb{R}$ and $x^{\prime}$ from $-R$ to $R$, we obtain

$$
A_{1} \leq \frac{C}{\sqrt{R}}
$$

This completes the proof of Theorem 1.1.

\section{Proof of Theorem 1.2}

We begin with the following result that ensures tightness.

Proposition 4.1. Let $u(t, x)$ be the solution to equation (1.1). Then for any $0 \leq s<t \leq T$ and any $p \geq 1$ there exists a constant $C=C(p, T)$ such that

$$
\mathbb{E}\left(\left|\int_{-R}^{R} u(t, x) d x-\int_{-R}^{R} u(s, x) d x\right|^{p}\right) \leq C R^{\frac{p}{2}}(t-s)^{\frac{p}{2}} .
$$

Proof. Let us assume that $s<t$. Recall that

$$
u(t, x)=1+\int_{0}^{t} \int_{\mathbb{R}} p_{t-r}(x-y) \sigma(u(r, y)) W(d r, d y),
$$

and thus

$$
\begin{aligned}
& \int_{-R}^{R} u(t, x) d x-\int_{-R}^{R} u(s, x) d x \\
& \quad=\int_{0}^{T} \int_{\mathbb{R}} \int_{-R}^{R} d x\left(p_{t-r}(x-y) \mathbf{1}_{\{r \leq t\}}-p_{s-r}(x-y) \mathbf{1}_{\{r \leq s\}}\right) \sigma(u(r, y)) W(d r, d y) .
\end{aligned}
$$

Moreover, recall that $\mathbb{E}\left(|u(s, y)|^{p}\right)$ is bounded on $s \leq T$ and $y \in \mathbb{R}$ for any $p \geq 1$. Using Burkholder-Davis-Gundy inequality, we can write

$$
\begin{aligned}
& \mathbb{E}\left(\left|\int_{-R}^{R} u(t, x) d x-\int_{-R}^{R} u(s, x) d x\right|^{p}\right) \\
\leq & c_{p} \mathbb{E}\left(\int_{0}^{T} \int_{\mathbb{R}}\left(\int_{-R}^{R}\left(p_{t-r}(x-y) \mathbf{1}_{\{r \leq t\}}-p_{s-r}(x-y) \mathbf{1}_{\{r \leq s\}}\right) d x\right)^{2} \sigma(u(r, y))^{2} d y d r\right)^{\frac{p}{2}} \\
\leq & c_{p}\left(\int_{0}^{T} \int_{\mathbb{R}}\left(\int_{-R}^{R}\left(p_{t-r}(x-y) \mathbf{1}_{\{r \leq t\}}-p_{s-r}(x-y) \mathbf{1}_{\{r \leq s\}}\right) d x\right)^{2}\|\sigma(u(r, y))\|_{p}^{2} d y d r\right)^{\frac{p}{2}} \\
\leq & C_{p, T}\left(\int_{0}^{T} \int_{\mathbb{R}}\left(\int_{-R}^{R}\left(p_{t-r}(x-y) \mathbf{1}_{\{r \leq t\}}-p_{s-r}(x-y) \mathbf{1}_{\{r \leq s\}}\right) d x\right)^{2} d y d r\right)^{\frac{p}{2}} .
\end{aligned}
$$


Thus it suffices to prove that

$$
\int_{0}^{T} \int_{\mathbb{R}}\left(\int_{-R}^{R}\left(p_{t-r}(x-y) \mathbf{1}_{\{r \leq t\}}-p_{s-r}(x-y) \mathbf{1}_{\{r \leq s\}}\right) d x\right)^{2} d y d r \leq C R(t-s) .
$$

Using Fourier transform we have

$$
\begin{aligned}
& \int_{0}^{T} \int_{\mathbb{R}}\left(\int_{-R}^{R}\left(p_{t-r}(x-y) \mathbf{1}_{\{r \leq t\}}-p_{s-r}(x-y) \mathbf{1}_{\{r \leq s\}}\right) d x\right)^{2} d y d r \\
= & C \int_{0}^{T} \int_{\mathbb{R}}\left(\int_{-R}^{R} e^{i \xi x} d x\right)^{2}\left(e^{-\frac{t-r}{2}|\xi|^{2}} \mathbf{1}_{\{r \leq t\}}-e^{-\frac{s-r}{2}|\xi|^{2}} \mathbf{1}_{\{r \leq s\}}\right)^{2} d \xi d r \\
= & C \int_{0}^{T} \int_{\mathbb{R}} \frac{\sin ^{2}(R|\xi|)}{|\xi|^{2}}\left(e^{-\frac{t-r}{2}|\xi|^{2}} \mathbf{1}_{\{r \leq t\}}-e^{-\frac{s-r}{2}|\xi|^{2}} \mathbf{1}_{\{r \leq s\}}\right)^{2} d \xi d r \\
= & C \int_{0}^{s} \int_{\mathbb{R}}\left(e^{-\frac{t-r}{2}|\xi|^{2}}-e^{-\frac{s-r}{2}|\xi|^{2}}\right)^{2} \frac{\sin ^{2}(R|\xi|)}{|\xi|^{2}} d \xi d r \\
& +C \int_{s}^{t} \int_{\mathbb{R}} e^{-(t-r)|\xi|^{2}} \frac{\sin ^{2}(R|\xi|)}{|\xi|^{2}} d \xi d r \\
:= & C\left(I_{1}+I_{2}\right) .
\end{aligned}
$$

For $I_{1}$ we can write

$$
\begin{aligned}
I_{1} & =\int_{0}^{s} \int_{\mathbb{R}} e^{r|\xi|^{2}}\left(e^{-\frac{t}{2}|\xi|^{2}}-e^{-\frac{s}{2}|\xi|^{2}}\right)^{2} \frac{\sin ^{2}(R|\xi|)}{|\xi|^{2}} d \xi d r \\
& =\int_{\mathbb{R}} \frac{1-e^{-s|\xi|^{2}}}{|\xi|^{2}}\left(e^{-\frac{t-s}{2}|\xi|^{2}}-1\right)^{2} \frac{\sin ^{2}(R|\xi|)}{|\xi|^{2}} d \xi .
\end{aligned}
$$

Using the bound $\left|1-e^{-a}\right| \leq \sqrt{a}$ for all $a \geq 0$ in the above parenthesis, we obtain that

$$
I_{1} \leq \int_{\mathbb{R}} \frac{1}{|\xi|^{2}}|\xi|^{2}|t-s| \frac{\sin ^{2}(R|\xi|)}{|\xi|^{2}} d \xi \leq C R|t-s|
$$

For $I_{2}$, using the bound $1-e^{-a} \leq a$ for any $a \geq 0$,

$$
I_{2}=\int_{\mathbb{R}} \frac{1-e^{-(t-s)|\xi|^{2}}}{|\xi|^{2}} \frac{\sin ^{2}(R|\xi|)}{|\xi|^{2}} d \xi \leq(t-s) \int_{\mathbb{R}} \frac{\sin ^{2}(R|\xi|)}{|\xi|^{2}} d \xi=C R(t-s) .
$$

The proof is finished by combining $I_{1}$ and $I_{2}$.

Proof of Theorem 1.2. It suffices to prove the convergence of the finite-dimensional distributions and tightness. However, the latter follows directly from Proposition 4.1 . 
In order to show the convergence of the finite-dimensional distributions, fix points $0 \leq t_{1}<\cdots<t_{m} \leq T$ and consider the random variables

$$
F_{R}^{(i)}=\frac{1}{\sqrt{R}}\left(\int_{-R}^{R} u\left(t_{i}, x\right) d x-2 R\right)
$$

for $i=1, \ldots, m$. We can write $F_{R}^{(i)}=\delta\left(v_{R}^{(i)}\right)$, where

$$
v_{R}^{(i)}(s, y)=\mathbf{1}_{\left[0, t_{i}\right]}(s) \frac{1}{\sqrt{R}} \int_{-R}^{R} p_{t_{i}-s}(x-y) \sigma(u(s, y)) d x .
$$

Set $F_{R}=\left(F_{R}^{(1)}, \ldots, F_{R}^{(m)}\right)$ and let $Z$ be an $m$-dimensional Gaussian centered vector with covariance

$$
C_{i, j}:=\mathbb{E}\left[Z^{i} Z^{j}\right]=\int_{0}^{t_{i} \wedge t_{j}} \xi(r) d r
$$

where we recall that $\xi(r)=\mathbb{E}\left[\sigma(u(r, x))^{2}\right]$. Then, applying Proposition 2.3 , for any $C^{2}$ function $h: \mathbb{R}^{m} \rightarrow \mathbb{R}$ with bounded second partial derivatives, we have

$$
\left|\mathbb{E}\left(h\left(F_{R}\right)\right)-\mathbb{E}(h(Z))\right| \leq \frac{1}{2}\left\|h^{\prime \prime}\right\|_{\infty} \sqrt{\sum_{i, j=1}^{m} \mathbb{E}\left[\left(C_{i, j}-\left\langle D F_{R}^{(i)}, v_{R}^{(j)}\right\rangle_{\mathfrak{H}}\right)^{2}\right]} .
$$

Then, it suffices to show that for each $i, j,\left\langle D F_{R}^{(i)}, v_{R}^{(j)}\right\rangle_{\mathfrak{H}}$ converges in $L^{2}$, as $R$ tends to infinity to $C_{i, j}$. To be more precisely, similarly with (3.3), we have

$$
\begin{aligned}
& \left\langle D F_{R}^{(i)}, v_{R}^{(j)}\right\rangle_{\mathfrak{H}} \\
= & \frac{1}{R} \int_{0}^{t_{i} \wedge t_{j}} \int_{\mathbb{R}} \int_{-R}^{R} \int_{-R}^{R} p_{t_{i}-s}(x-y) p_{t_{j}-s}(\tilde{x}-y) \sigma^{2}(u(s, y)) d x d \tilde{x} d y d s \\
& +\frac{1}{R} \int_{0}^{t_{i} \wedge t_{j}} \int_{\mathbb{R}} \int_{-R}^{R} \int_{-R}^{R} p_{t_{j}-s}(\tilde{x}-y) \sigma(u(s, y)) \\
& \times\left(\int_{s}^{t_{i}} \int_{\mathbb{R}} p_{t_{i}-r}(x-z) \Sigma(r, z) D_{s, y} u(r, z) W(d r, d z)\right) d x d \tilde{x} d y d s \\
:= & I_{1, i, j}(R)+I_{2, i, j}(R) .
\end{aligned}
$$

Then we obtain that

$$
\mathbb{E}\left[\left(C_{i j}-\left\langle D F_{R}^{(i)}, v_{R}^{(j)}\right\rangle_{\mathfrak{H}}\right)^{2}\right] \leq 2 \mathbb{E}\left(C_{i j}-I_{1, i, j}(R)\right)^{2}+2 \mathbb{E}\left(I_{2, i, j}(R)^{2}\right) .
$$

By noting that

$$
C_{i j}=\lim _{R \rightarrow \infty} \mathbb{E}\left(I_{1, i, j}(R)\right),
$$


and using arguments similar as those in the proof of Theorem 1.1. we can show that $\mathbb{E}\left[\left(C_{i j}-\left\langle D F_{R}^{(i)}, v_{R}^{(j)}\right\rangle_{\mathfrak{H}}\right)^{2}\right] \rightarrow 0$ as $R \rightarrow \infty$. The proof is finished.

\section{Appendix}

Here we prove a technical lemma which is used in the proof of Theorem 1.1.

Lemma 5.1. Let $\left\|D_{s, y} u(r, z)\right\|_{p}^{2}$ satisfy

$$
\left\|D_{s, y} u(r, z)\right\|_{p}^{2} \leq C p_{r-s}^{2}(z-y)+C \int_{s}^{r} \int_{\mathbb{R}} p_{r-r_{1}}^{2}\left(z-z_{1}\right)\left\|D_{s, y} u\left(r_{1}, z_{1}\right)\right\|_{p}^{2} d z_{1} d r_{1}
$$

for any $0<s<r \leq t$ and $y, z \in \mathbb{R}$. Then we have

$$
\left\|D_{s, y} u(r, z)\right\|_{p} \leq C_{t, p} p_{r-s}(z-y) .
$$

for some constant $C_{t, p}$ which depends on $t$ and $p$.

Proof. By iterating (5.1) we have

$$
\begin{aligned}
& \left\|D_{s, y} u(r, z)\right\|_{p}^{2} \\
& \leq C p_{r-s}^{2}(z-y)+C^{2} \int_{s}^{r} \int_{\mathbb{R}} p_{r-r_{1}}^{2}\left(z-z_{1}\right) p_{r_{1}-s}^{2}\left(z_{1}-y\right) d z_{1} d r_{1} \\
& +\cdots+C^{n} \int_{s}^{r} \int_{\mathbb{R}} \int_{s}^{r_{1}} \int_{\mathbb{R}} \cdots \int_{s}^{r_{n-1}} \int_{\mathbb{R}} p_{r-r_{1}}^{2}\left(z-z_{1}\right) p_{r_{1}-r_{2}}^{2}\left(z_{1}-z_{2}\right) \times \cdots \\
& \quad \times p_{r_{n-1}-r_{n}}^{2}\left(z_{n-1}-z_{n}\right) p_{r_{n}-s}^{2}\left(z_{n}-y\right) d z_{n} d r_{n} \cdots d z_{1} d r_{1} \\
& +\int_{s}^{r} \int_{\mathbb{R}} \int_{s}^{r_{1}} \int_{\mathbb{R}} \cdots \int_{s}^{r_{n}} \int_{\mathbb{R}} p_{r-r_{1}}^{2}\left(z-z_{1}\right) p_{r_{1}-r_{2}}^{2}\left(z_{1}-z_{2}\right) \times \cdots \\
& \quad \times p_{r_{n-1}-r_{n}}^{2}\left(z_{n-1}-z_{n}\right) p_{r_{n}-r_{n+1}}^{2}\left(z_{n}-z_{n+1}\right) \\
& \quad \times\left\|D_{s, y} u\left(r_{n+1}, z_{n+1}\right)\right\|_{p}^{2} d z_{n+1} d r_{n+1} \cdots d z_{1} d r_{1} .
\end{aligned}
$$

Then, using the fact that

$$
p_{r-s}^{2}(z)=\frac{C}{\sqrt{r-s}} p_{\frac{r-s}{2}}(z),
$$

and

$$
\int_{\mathbb{R}} p_{s}(x-y) p_{t}(y-z) d y=p_{s+t}(x-z),
$$


we have for the integrals of the product of heat kernels above,

$$
\begin{aligned}
& \int_{s}^{r} \int_{\mathbb{R}} \int_{s}^{r_{1}} \int_{\mathbb{R}} \cdots \int_{s}^{r_{n-1}} \int_{\mathbb{R}} p_{r-r_{1}}^{2}\left(z-z_{1}\right) p_{r_{1}-r_{2}}^{2}\left(z_{1}-z_{2}\right) \times \cdots \\
& \times p_{r_{n-1}-r_{n}}^{2}\left(z_{n-1}-z_{n}\right) p_{r_{n}-s}^{2}\left(z_{n}-y\right) d z_{n} d r_{n} \cdots d z_{1} d r_{1} \\
= & C^{n} \int_{s}^{r} \int_{s}^{r_{1}} \cdots \int_{s}^{r_{n-1}} \int_{\mathbb{R}^{n}} \frac{1}{\sqrt{r-r_{1}}} \frac{1}{\sqrt{r_{1}-r_{2}}} \cdots \frac{1}{\sqrt{r_{n-1}-r_{n}}} \frac{1}{\sqrt{r_{n}-s}} \\
& \times p_{\frac{r-r_{1}}{2}}\left(z-z_{1}\right) p_{\frac{r_{1}-r_{2}}{2}}\left(z_{1}-z_{2}\right) \times \cdots \times p_{\frac{r_{n-1}-r_{n}}{2}}\left(z_{n-1}-z_{n}\right) p_{\frac{r_{n}-s}{2}}\left(z_{n}-y\right) \\
& \times d z_{n} \cdots d z_{1} d r_{n} \cdots d r_{1} \\
= & C^{n} \int_{s}^{r} \int_{s}^{r_{1}} \cdots \int_{s}^{r_{n-1}} \frac{1}{\sqrt{r-r_{1}}} \frac{1}{\sqrt{r_{1}-r_{2}}} \cdots \frac{1}{\sqrt{r_{n-1}-r_{n}}} \frac{1}{\sqrt{r_{n}-s}} p_{\frac{r-s}{2}}(z-y) d r_{n} \cdots d r_{1} \\
= & (r-s)^{\frac{n-1}{2}} \frac{\Gamma\left(\frac{1}{2}\right)^{n+1}}{\Gamma\left(\frac{n+1}{2}\right)} p_{\frac{r-s}{2}}(z-y) \\
= & (r-s)^{\frac{n}{2}} \frac{\Gamma\left(\frac{1}{2}\right)^{n+1}}{\Gamma\left(\frac{n+1}{2}\right)} p_{r-s}^{2}(z-y) .
\end{aligned}
$$

Thus we obtain

$$
\begin{aligned}
& \left\|D_{s, y} u(r, z)\right\|_{p}^{2} \leq\left(\sum_{j=0}^{n} C^{j}(r-s)^{\frac{j}{2}} \frac{\Gamma\left(\frac{1}{2}\right)^{j+1}}{\Gamma\left(\frac{j+1}{2}\right)}\right) p_{r-s}^{2}(z-y) \\
& +C^{n}(r-s)^{\frac{n}{2}} \frac{\Gamma\left(\frac{1}{2}\right)^{n+1}}{\Gamma\left(\frac{n+1}{2}\right)} \\
& \quad \times \int_{s}^{r} \int_{\mathbb{R}} p_{r-r_{n+1}}^{2}\left(z-z_{n+1}\right)\left\|D_{s, y} u\left(r_{n+1}, z_{n+1}\right)\right\|_{p}^{2} d z_{n+1} d r_{n+1} .
\end{aligned}
$$

Taking into account that the first term on the right-hand side is a convergent series and the second term tends to 0 as $n \rightarrow \infty$, the proof is finished.

\section{References}

[1] L. Chen, Y. Hu and D. Nualart: Regularity and strict positivity of densities for the nonlinear stochastic heat equation. Mem. Amer. Math. Soc. To appear.

[2] L. Chen, D. Khoshnevisan and K. Kim: Decorrelation of total mass via energy. Potential Analysis 45, (2016), 157-166.

[3] D. Conus, M. Joseph and D. Khoshenvisan: On the chaotic character of the stochastic heat equation, before the onset of intermitttency. Ann. Probab. 41 (2013), no. 3B, 2225-2260.

[4] D. Conus, M. Joseph, D. Khoshenvisan and S. Shiu: On the chaotic character of the stochastic heat equation, II Probab. Theory Related Fields. 156 (2013), no. 3-4, 483-533. 
[5] B. Gaveau and P. Trauber: L'intégrale stochastique comme opérateur de divergence dans l'espace founctionnel. J. Funct. Anal. 46 (1982), 230-238.

[6] I. Nourdin and G. Peccati: Normal approximations with Malliavin calculus. From Stein's method to universality. Cambridge Tracts in Mathematics, 192. Cambridge University Press, Cambridge, 2012. xiv +239 pp.

[7] D. Nualart: The Malliavin calculus and related topics. Second edition. Probability and its Applications (New York). Springer-Verlag, Berlin, 2006. xiv +382 pp.

[8] D. Nualart and E. Pardoux: Stochastic calculus with anticipating integrands. Probab. Theory Re. Fields 78 (1988), 535-581.

[9] D. Nualart and L. Quer-Sardanyons: Existence and smoothness of the density for spatially homogeneous SPDEs. Potential Analysis 27 (2007), 281-299.

[10] Walsh, John B. An Introduction to Stochastic Partial Differential Equations. In: Ècole d'èté de probabilités de Saint-Flour, XIV-1984, 265-439. Lecture Notes in Math. 1180, Springer, Berlin, 1986.

University of Birmingham, School of Mathematics, UK

E-mail address: j.huang.4@bham.ac.uk

University of Kansas, Department of Mathematics, USA

E-mail address: nualart@ku.edu

University of Helsinki, Department of Mathematics and Statistics, Finland

E-mail address: lauri.viitasaari@iki.fi 